\title{
Gemeinsam engagiert
}

\section{Praktische Erfahrungen mit Corporate Citizenship in Mitteldeutschland}

\author{
Ellen Sturm und Olaf Ebert
}

Ellen Sturm ist Leiterin des Projektes »Corporate Citizenship - gemeinsam engagiert « der Freiwilligen-Agentur Halle-Saalkreis e. V.

E-Mail ellen.sturm@freiwilligen-agentur.de

Olaf Ebert ist Geschäftsführer der Freiwilligen-Agentur Halle-Saalkreis e. V., Vorsitzender der

Landesarbeitsgemeinschaft der Freiwilligenagenturen Sachsen-Anhalt e. V. und Kuratoriumsmitglied der Bürgerstiftung Halle.

E-Mail olaf.ebert@freiwilligen-agentur.de
Eine gezielte Anbahnung und Entwicklung von Kooperationsprozessen zwischen Wirtschaft und drittem Sektor braucht förderliche Rabmenbedingungen. Die Erfabrungen eines Projektes in Sachsen-Anhalt zeigen, wie gemeinsames Engagement von Unternehmen und gemeinnützigen Organisationen erreicht werden kann.

„Corporate Citizenship - gemeinsam engagiert " - unter diesem Motto hat die Freiwilligen-Agentur Halle-Saalkreis e. V. mit dem gleichnamigen Modellprojekt 2005 damit begonnen, neue Kooperationen zwischen Wirtschaftsunternehmen und gemeinnützigen Organisationen in Halle und Umgebung zu initiieren und Kompetenzen und Ressourcen verlässlicher Partnerschaften jenseits von Markt und Staat zusammenzuführen. Aufgabe des Projektes ist es, nachhaltig wirksame und übertragbare Methoden zur Förderung neuer Partnerschaften zwischen dem Profit- und dem Non-Profit-Bereich zu entwickeln und zu erproben. Mehr als 30 Kooperationsprojekte sind aus der zweieinhalbjährigen Projektlaufzeit hervorgegangen.

Kern des Projektes und gleichzeitig Ausgangspunkt für viele Kooperationsprojekte zwischen Unternehmen und gemeinnützigen Organisationen bildet der Wettbewerb »engagiert für halle«. Mit diesem Preis werden nachahmenswerte und beispielgebende Projekte ausgezeichnet, die durch das Engagement der halleschen Bürgerinnen und Bürger realisiert werden (vgl. Kasten »Kriterien für den Wettbewerb sengagiert für halle«). Bundesweit einzigartig ist dabei die nachhaltige Unterstützung der Preisträgerprojekte innerhalb einer einjährigen Unternehmenspartnerschaft.

In den Partnerschaften gibt es viele Anknüpfungspunkte für eine gemeinsame Arbeit und häufig wird die Zusammenarbeit über das eine geplante Jahr hinaus fortgeführt, wie das Beispiel des Kooperationsprojektes des kommunalen Ener- gieversorgungsunternehmens EVH mit dem Mehrgenerationenhaus Halle zeigt. So spendete die EVH GmbH u. a. einen Computerarbeitsplatz, schulte die pädagogischen Mitarbeiterinnen und Mitarbeiter zum Thema Kundenbetreuung und unterstützte das Mehrgenerationenhaus in der Öffentlichkeitsarbeit sowie bei der Organisation einer Ausstellung. Im Gegenzug nutzte das Unternehmen Veranstaltungsangebote des Mehrgenerationenhauses für pensionierte Mitarbeiterinnen und Mitarbeiter. Darüber hinaus erhielten die Beschäftigten die Möglichkeit, eine flexible Kinderbetreuung in Anspruch zu nehmen.

\section{Unternehmensengagement in der Region Halle}

Kooperationsprojekte zwischen Profitund Non-Profit-Bereich in der Region Halle sind immer noch selten und entstehen oft zufällig. Es bestehen dementsprechend vielfältige, noch ungenutzte Möglichkeiten für Unternehmen, sich an Projekten im Gemeinwesen zu beteiligen. Die Ergebnisse einer projektbegleitenden Studie zum gesellschaftlichen Engagement von Unternehmen zeigen: Es gibt eine große Bereitschaft von Unternehmen am Wirtschaftsstandort Halle, sich gesellschaftlich zu engagieren. Diese Erkenntnisse stimmen in vielen Punkten mit den Befunden anderer Studien zum Thema Corporate Citizenship in Deutschland überein (vgl. Freiwilligen-Agentur Halle-Saalkreis e. V. 2007, S. 23 ff.).

Für viele Unternehmen ist das gesellschaftliche Engagement im direkten regionalen Umfeld beispielsweise durch Geldund Sachspenden selbstverständlich und erfolgt zumeist mit einer gewissen Kontinuität. Gleichwohl zeigen sich im gesellschaftlichen Engagement von Unternehmen unausgeschöpfte Potenziale. Sowohl die Untersuchungsergebnisse als auch die Erfahrungen des Projektes lassen den Schluss zu, dass es bei den Unternehmen 


\section{Kooperationsvereinbarung}

\section{zwischen dem Unternehmen:}

Name:

Anschrift:

Telefon:

E-Mail:

\section{und der gemeinnützigen Einrichtung:}

Name:

Anschrift:

Telefon:

E-Mail:

Die Kooperationspartner beabsichtigen im Rahmen einer Partnerschaft im Jahr.

/ für das

Projekt zusammenzuarbeiten

Im Rahmen dieser Partnerschaft unterstützt das Unternehmen die gemeinnützige Einrichtung in folgenden Bereichen:

-

Im Gegenzug unterstützt die gemeinnützige Einrichtung das Unternehmen in folgenden Aktivitäten:

\section{$\bullet$}

$\cdot$

-

Jeder Kooperationspartner unterrichtet den jeweils anderen umgehend über alle Umstände, welche für die Durchführung dieser Vereinbarung von Bedeutung sein könnten. Maßnahmen, die die Öffentlichkeitswirkung des jeweils anderen berühren, werden zuvor mit dem Kooperationspartner abgestimmt.

Ort, Datum

Für das Unternehmen:

Für die gemeinnützige Einrichtung: 
erhebliche Handlungsspielräume, Innovationspotenziale und Verbesserungsmöglichkeiten gibt; vor allem bei

- der Auswahl von Themen und Feldern ihres gesellschaftlichen Engagements,

- der Entwicklung und dem Einsatz von geeigneten Instrumenten,

- der Recherche und Entscheidung über gemeinnützige Kooperationspartner

- und beim Kontakt und Austausch mit anderen gesellschaftlich engagierten Unternehmen.

Hier bestehen für Unternehmen in der Kooperation mit regionalen Vermittlern vielversprechende Optionen. Inzwischen hat sich die Freiwilligen-Agentur HalleSaalkreis e. V. zu einem Mittler für Unternehmenskooperationen entwickelt und bildet eine Drehscheibe für Corporate-Citizenship-Aktivitäten in der Region, indem sie Unternehmen bei der Auswahl passender gemeinnütziger Organisationen berät und sie und gemeinnützige Organisationen bei der Vorbereitung, Begleitung und Evaluation ihrer Engagementprojekte unterstützt sowie Netzwerke und Dialogforen auf regionaler Ebene initiiert.

\section{Anforderungen und Perspektiven}

Ein Rückblick auf die letzten zehn Jahre lässt eine Entwicklung sowohl in der Debatte über das gesellschaftliche Engagement von Unternehmen als auch bei der Umsetzung von konkreten Maßnahmen und Strategien in Deutschland erkennen. Diese Entwicklungen sind nicht zuletzt auf verschiedene Aktivitäten einzelner Organi- sationen, Projekte und Initiativen zurückzuführen, die in diesem Feld aktiv sind und versuchen, neue Kooperationsmodelle zwischen Unternehmen und gemeinnützigen Organisationen anzuregen und zu fördern (vgl. Bertelsmann Stiftung 2008).

Auch in der Region Halle ist in den letzten Jahren eine positive Entwicklung in diesem Bereich zu beobachten. So hat sich bei den verschiedenen Akteuren ein Verständnis für die Chancen und Möglichkeiten von Corporate Citizenship entwickelt. Dies ist mitunter auf die Schaffung eines aktivierenden Umfelds zurückzuführen. Allerdings ist das Aktivierungspotenzial längst noch nicht ausgeschöpft. An dieser Stelle gilt es, gemeinsam mit anderen Partnern das Thema Corporate Citizenship in der Region noch stärker zu etablieren und Orte zu schaffen, die den unterschiedlichen Beteiligten Raum für Begegnung und Austausch bieten, Netzwerke anregen und das Thema in die Öffentlichkeit transportieren.

Entwicklungspotenzial liegt auch in der Aktivierung weiterer Unternehmen, die bislang noch nicht die Bedeutung und Möglichkeiten von Corporate Citizenship wahrgenommen haben. Ein zunehmendes Interesse für lokale Belange ist deutlich spürbar und gewinnt für Unternehmen an Relevanz. Ziel wird es sein, das gesellschaftliche Engagement von Unternehmen in Zukunft verstärkt zu würdigen, sichtbar zu machen und weiterzuentwickeln.

Um die eigenen Kooperationspotenziale mit Unternehmen besser zu erkennen und die Ressourcen und Kompetenzen engagierter Unternehmen zur Ergänzung

\section{Kriterien für den Wettbewerb »engagiert für halle«}

Einbindung, Förderung von Ehrenamtlichen:

- Wie (sehr) bindet das Projekt ehrenamtliche Helferinnen und Helfer ein?

- Inwieweit motiviert das Projekt zu ehrenamtlichen Tätigkeiten?

Beachtung des Mottos für den Standort Halle:

- Inwieweit engagiert sich das Projekt für den Standort Halle?

- Trifft es den Bedarf bzw. die spezielle Situation in Halle?

Wirksamkeit, Effizienz, Nachhaltigkeit:

- Sind die Ziele mit den vorgeschlagenen Mitteln erreichbar?

- Ist der Aufwand den Zielen angemessen?

- Ist das Projekt auf lange Frist angelegt?

Offenheit für Partnerschaften:

- Ist das Projekt bereit, in eine Partnerschaft zu »investieren«?

- Gibt es bereits Ideen oder Erfahrungen für eine Unternehmenspartnerschaft? ihrer fachlichen Arbeit besser einbinden zu können, bedarf es in den gemeinnützigen Organisationen einer Professionalisierung. Das Interesse an Fortbildungen in diesem Bereich lässt auf weitere Unterstützungsbedarfe bei den gemeinnützigen Organisationen schließen.

Die Kommune unterstützt gesellschaftliches Engagement von Unternehmen derzeit vor allem durch die Schaffung begünstigender Rahmenbedingungen, beispielsweise durch die Unterstützung des erwähnten Wettbewerbs »engagiert für halle« und überlässt es Mittlerorganisationen, Unternehmensnetzwerken und einzelnen Unternehmen, neue CorporateCitizenship-Aktivitäten anzuregen. Das Land Sachsen-Anhalt hat unter Berücksichtigung der besonderen Situation in Ostdeutschland ein hohes Interesse am Thema Corporate Citizenship und ist spätestens hier gefordert, die vorhandenen Ansätze zu stärken und unterstützende Rahmenbedingungen des gesellschaftlichen Engagements von Unternehmen politisch zu gestalten.

Sicherlich stellen der Ausbau und die Weiterentwicklung von langfristigen Unternehmenspartnerschaften und der Aufbau von Kooperationsstrukturen an alle Akteure hohe Anforderungen. Wenn es jedoch gelingt, geeignete Rahmenbedingungen zu schaffen, wächst die Chance für einen nachhaltigen Aufbau gemeinsam getragener Verantwortung.

\section{Literatur}

Bertelsmann Stiftung (Hg.) 2008: Grenzgänger, Pfadfinder, Arrangeure. Mittlerorganisationen zwischen Unternehmen und Gemeinwohlorganisationen. Gütersloh.

Freiwilligen-Agentur Halle-Saalkreis e. V. (Hg.) 2007: Praxishandbuch »Corporate Citizenship - gemeinsam engagiert«. Erfahrungen, Strategien und Modelle für gesellschaftliches Engagement von Unternehmen in Mitteldeutschland. Halle. Das Handbuch steht im Internet kostenlos zur Verfügung: http://www.gemeinsam-engagiert.de. 